PROCEEDINGS OF THE

AMERICAN MATHEMATICAL SOCIETY

Volume 33, Number 1, May 1972

\title{
BAND DECOMPOSITIONS OF SEMIGROUPS
}

\author{
MOHAN S. PUTCHA AND JULIAN WEISSGLASS
}

Abstract. The existence of a band decomposition of a semigroup into semigroups having at most one idernpotent and a group idea! whenever it has an idempotent is investigated. It is shown that $S$ has such a band decomposition if and only if, for every $c, d \in S^{1}$, $a \in S$, the idempotent multiples of $c a d$ and $c a^{2} d$ coincide. The main result is used to characterize those semigroups which are bands of groups, extending a theorem of Clifford.

1. Introduction. The primary purpose of this paper is to investigate band decompositions of semigroups into semigroups having at most one idempotent and a group ideal whenever it has an idempotent. One result of this investigation is a characterization of those semigroups which are bands of groups, extending a theorem of Clifford (cf. [1] or [2, §4.2, Exercise 3]).

Throughout this paper $S$ will denote a semigroup and $E=E(S)$ the set of idempotents of $S$. The set-valued function $\mathfrak{W}$ on $S$ is defined by $\mathfrak{B}(a, E(S))=$ $S a \cap a S \cap E . \mathfrak{M B}(a, E(S))$ can be thought of as the set of idempotent multiples of $a$ in $S$. We shall write $\mathfrak{M}(a)$ for $\mathfrak{M}(a, E(S))$ when there is no possibility of confision. Any terms which are not defined in this article can be found in Clifford and Preston [2].

The concept of a viable semigroup was introduced in [3]. $S$ is viable if $a b, b a \in E$ implies $a b=b a$. We proved in [3] that $S$ is viable if and only if $S$ is a semilattice of $\mathscr{S}$-indecomposable semigroups each of which contains at most one idempotent and a group ideal whenever it has an idempotent.

A semigroup $S$ is weakly viable if for every $c, d \in S^{1}, a \in S, \mathfrak{B}(c a d)=$ $\mathfrak{B}\left(c a^{2} d\right)$. That a viable semigroup is weakly viable follows from the corollary to Theorem 6 .

The main theorem (Theorem 6) of this paper proves, in part, that $S$ is weakly viable if and only if it is a band of semigroups having at most one idempotent and a group ideal whenever it has an idempotent. As a consequence of this result, the structure of certain weakly viable semigroups is determined. For example, Theorem 7 proves that $S$ is weakly

Received by the editors April 26, 1971.

AMS 1969 subject classifications. Primary 2093.

Key rords aná phrases. Senigroups, band, group-ideals, nil semigroups.

if American Mathematical Society 1972 
viable and some power of each element lies in a subgroup of $S$ if and only if $S$ is a band of semigroups which are ideal extensions of groups by nil semigroups. Also, Theorem 9, the result referred to above, proves, in part, that $S$ is weakly viable, separative and some power of each element lies in a subgroup if and only if $S$ is a band of groups.

The final result of this paper indicates the extent of the class of weakly viable semigroups. In particular, viable semigroups and medial semigroups are weakly viable.

2. Decomposition theorems. In order to shorten our proofs we will say $a \mid b$ if $b \in S^{1} a \cap a S^{1}$. Then the set-valued function $\mathfrak{W}$ on $S$, defined above, is given by $\mathfrak{B}(a)=\{e|e \in E, a| e\}$.

Define the congruence relation $\beta$ on $S$ by $a \beta b$ if and only if $\mathfrak{B}(\mathrm{cad})=$ $\mathfrak{W}(c b d)$ for every $c, d \in S^{\mathbf{1}}$.

THEOREM 1. Let $S$ be weakly viable. Then:

(i) $\beta$ is a congruence relation on $S$ and $S / \beta$ is a band.

(ii) Each $\beta$-class contains at most one idempotent and a group ideal whenever it contains an idempotent.

(iii) If $\beta^{\prime}$ is any other congruence on $S$ satisfying (i) and (ii) then $\beta^{\prime} \subseteq \beta$.

(iv) $\mathscr{H} \subseteq \beta$. In fact, $\mathscr{H}$ is contained in any congruence $\beta$ such that $S ! \beta$ is a band.

(v) If $e \in \mathfrak{W}(a)$, then ae $\beta$ e $\beta$ ea.

Proof. (i) Suppose $a \beta b$ and $s \in S$. Let $c, d \in S^{1}$. Then $\mathfrak{M}($ casd $)=$ $\mathfrak{W}(c a(s d))=\mathfrak{W}(c b s d)$. Hence as $\beta$ bs. Similarly $s a \beta s b$ and hence $\beta$ is a congruence. From the definition of weakly viable, $\mathfrak{B}(c a d)=\mathfrak{W}\left(c a^{2} d\right)$. Hence $a \beta a^{2}$ and $S / \beta$ is a band.

(ii) Suppose $e_{1} \beta e_{2}$. Then $\mathfrak{W}\left(c e_{1} d\right)=\mathfrak{W}\left(c e_{2} d\right)$, for all $c$, $d \in S^{1}$. In particular, $\mathfrak{W}\left(e_{1}\right)=\mathfrak{W}\left(e_{2}\right)$. Since $e_{i} \in \mathfrak{W}\left(e_{i}\right), i=1,2$, we have $e_{2} \mid e_{1}$ and $e_{1} \mid e_{2}$. Thus $e_{1} \mathscr{H} e_{2}$ and by [2, Lemma 2.15], $e_{1}=e_{2}$. Hence each $\beta$-class contains at most one idempotent. Let $B$ be a $\beta$-class containing the idempotent $e$ and let $a \in B$. Then $e \in \mathfrak{W}(e)=\mathfrak{W}(a)=\mathfrak{W}\left(a^{2}\right)$. Hence there exists $x \in S$ such that $a^{2} x=e$. But $a \beta a^{2}$ implies $a x \beta a^{2} x=e$. Hence $a x \in B$ and $a(a x)=e$. Thus $e$ is a right zeroid of $B$. Similarly $e$ is a left zeroid and hence by [1, $\$ 2.5$, Exercise 6], $B$ has a group ideal.

(iii) Suppose $a \beta^{\prime} b$ and that $e \in \mathfrak{M}(c a d)$ with $c, d \in S^{1}$. Then cadle so there exists $x \in S$, such that $c a d x=e$. Hence $c b d x \beta^{\prime} e$. Since $e$ is in the group ideal of its $\beta^{\prime}$-class we have $c b d x \mid e$. Hence $c b d x x^{\prime}=e$, for some $x^{\prime} \in S$. Consequently $e \in c b d S$. Similarly $e \in S c b d$ and so $\mathfrak{B}(c a d) \subseteq \mathfrak{W}(c b d)$. By symmetry, $\mathfrak{W}(c b d) \subseteq \mathfrak{W}(c a d)$ and hence $\mathfrak{W}(c a d)=\mathfrak{W}(c b d)$. Therefore $a \beta b$.

(iv) Suppose $a \mathscr{H}^{\prime} b$. Then, in particular, there exist $x, y \in S^{1}$ such that $a=b x, b=y a$. Since $b \beta b^{2}, a \beta a^{2}$ and $\beta$ is a congruence we have $a=$ $b x \beta b^{2} x=b a=y a^{2} \beta y a=b$. Hence $a \beta b$. 
(v) Let $a x=e=y a$. Then $e a=y a^{2} \beta y a=e=a x \beta a^{2} x=a e$. Hence ea $\beta$ e $\beta$ ae.

COROLLARY. Let $S$ be a band of one idempotent semigroups with group ideals. Then this decomposition coincides with the $\beta$-decomposition of Theorem 1 and hence is unique.

Proof. It is easy to prove that $S$ is weakly viable (see Theorem 6). Let $\beta^{\prime}$ be the congruence induced by this decomposition. By Theorem 1(iii), $\beta^{\prime} \subseteq \beta$. Suppose $\alpha \beta b$. Since each component contains an idempotent there exist $e_{1}, e_{2} \in E$ such that $a \beta^{\prime} e_{1}, b \beta^{\prime} e_{2}$. But since $\beta^{\prime} \subseteq \beta, a \beta e_{1}$ and $b \beta e_{2}$. Hence $e_{1} \beta e_{2}$, which implies $e_{1}=e_{2}$. Consequently $a \beta^{\prime} b$.

LeMma 2. Let I be an idempotent free ideal of $S$. Then $S$ is weakly viable if and only if the Rees factor semigroup $S_{/} I$ is weakly viable.

Proof. Let 0 denote the equivalence class $I$ in $S / I$. Since $I$ is idempotent free $E(S / I)=E(S) \cup 0$. Let $e \in E(S)$. Since $I$ is an ideal, $x \mid e$ in $S$ if and only if $x \mid e$ in $S / I$. But for every $a \in S / 1, a \mid 0$. Hence $\mathfrak{B}(x, E(S)) \cup\{0\}=$ $\mathfrak{W}(x, E(S / I))$ for all $x \in S$. The result easily follows.

A semigroup $S$ is $E$-inversive if for every $a \in S$ there exists $x \in S$ such that $a x \in E$.

LEMMA 3. The following are equivalent.

(i) For every $a \in S, S^{1} a S^{1} \cap E \neq \varnothing$.

(ii) $S$ is E-inversive.

(iii) For every $a \in S, \mathfrak{W}(a) \neq \varnothing$.

Proof. (i) $\Rightarrow$ (ii). Let $a \in S$. There exist $s, t \in S^{1}$ such that $s a t \in E$. But then $a($ tsats $) \in E$. Hence $S$ is $E$-inversive.

(ii) $\Rightarrow$ (iii). Let $a \in S$. Then $a x \in E$ for some $x \in S$. Let $s=x a x$. Then $e_{1}=a s$ and $e_{2}=s a$ are idempotents. (This observation is due to Croisot. See [1, §3.2, Exercise 8].) There exists $y$ such that $e_{2} e_{1} y \in E$. Hence $e_{3}=e_{1} y e_{2} e_{1} y e_{2} \in E$ and $e_{1} e_{3}=e_{3}=e_{3} e_{2}$. Thus $a\left(s e_{3}\right)=e_{3}=\left(e_{3} s\right) a$, so that $a \mid e_{3} \in E$. Hence $\mathfrak{W}(a) \neq \varnothing$.

(iii) $\Rightarrow$ (i) a fortiori.

If $B$ is a $\beta$-class of $S$, define $\mathfrak{W}(B)=\mathfrak{W}(b)$ where $b$ is any element of $B$. The definition is independent of the choice of $b$ in $B$. If $s \in S$, let [s] denote the $\beta$-class of $s$.

Lemma 4. Let $S$ be neakly viable and $B$ a $\beta$-class of $S$. If $e \in \mathfrak{M}(B)$, then $B \cup[e]$ is a subsemigroup of $S$ having $[e]$ as an ideal and the group ideal of $[e]$ as a group ideal. Hence if $B$ is idempotent free, $B \cup[e]$ is a one idempotent semigroup with group ideai.

Proof. Let $x, y \in B \cup[e]$. If $x$ and $y$ are both in $B$ or both in $[e]$, then clearly $x y \in B \cup[e]$. So for $B \cup[e]$ to be a semigroup it suffices to prove that 
$y x, x y \in B \cup[e]$ whenever one is in $B$ and one is in [e]. Suppose $x \in B, y \in[e]$. Then $e \in \mathfrak{W}(x)$ so by Theorem $1(\mathrm{v})$, xe $\beta$ e $\beta$ ex. But $e \beta y$, so ex $\beta y x$ and $x e \beta x y$. Hence $x y, y x \in[e]$. This shows $B \cup[e]$ is a semigroup with $[e]$ as an ideal. Moreover, for every $a \in B \cup[e], a e, e a \in[e]$. Hence $e a, a e \mid e$ and $e$ is a zeroid. By $[2, \S 2.5$, Exercise 6], $B \cup[e]$ has a group ideal. It is easy to see that the group ideal of $B \cup[e]$ is in fact the group ideal of $[e]$.

Proposition 5. $S$ is weakly viable and E-inversive if and only if $S=$ $\bigcup\left\{S_{\alpha} \mid \alpha \in \Omega\right\}$ where each $S_{\alpha}$ is a one idempotent semigroup with group ideal and for each $a \in S, c, d \in S^{1}$, there exists $S_{\alpha}$ such that cad, $c a^{2} d \in S_{\alpha}$.

Proof. Assume $S$ is weakly viable and $E$-inversive. Let $\mathfrak{F}=\{B \mid B \in S / \beta$, $B$ is idempotent free $\}$. By Lemma $3, \mathfrak{M}(B) \neq \varnothing$, so by the axiom of choice there exists a function $f: \mathfrak{F} \rightarrow E$, such that $f(B) \in \mathfrak{W}(B)$. Define

$$
\begin{aligned}
S_{B} & =B & & \text { if } B \text { has an idempotent, } \\
& =B \cup[f(B)] & & \text { otherwise. }
\end{aligned}
$$

Then clearly $S=\bigcup\left\{S_{B}|B \in S| \beta\right\}$ and each $S_{B}$ has exactly one idempotent. That each $S_{B}$ has a group ideal follows from Lemma 4 . Now let $a \in S$; $c, d \in S^{1}$. Then $c a d, c a^{2} d$ belong to the same $\beta$-class, say $B$, and hence $c a d$, $c a^{2} d \in B \subseteq S_{B}$.

Conversely, let $e \in E$. If $e \in \mathfrak{W}(c a d)$ then there exists $x \in S$ such that $c a d x=e$. By hypothesis $c a^{2} d x$ and $c a d x=e$ belong to the same $S_{\alpha}$. So $e$ must be the idempotent of the group ideal of $S_{x}$. Hence there exists $y \in S_{\alpha}$ such that $c a^{2} d x y=e$. Hence $e \in c a^{2} d S$. Similarly $e \in S c a^{2} d$. Hence $e \in \mathfrak{M}\left(c a^{2} d\right)$. A similar argument proves that $\mathfrak{W}\left(c a^{2} d\right) \subseteq \mathfrak{W}(c a d)$ and we conclude that $S$ is weakly viable. That $S$ is $E$-inversive is immediate.

REMARK. A proof similar to that above proves that $S$ is viable and $\Sigma$-inversive if and only if $S=\bigcup\left\{S_{\alpha} \mid \alpha \in \Omega\right\}$ where each $S_{\alpha}$ is a one idempotent semigroup with group ideal and such that for all $a, b \in S$, there exist $S_{\alpha}$ such that $a b, b a \in S_{\alpha}$.

THEOREM 6. The following are equivalent.

(i) $S$ is weakly viable.

(ii) $S$ is a band of semigroups having at most one idempotent and a group ideal whenever it has an idempotent.

(iii) $S$ is an ideal extension of an idempotent free semigroup (possibly empty) by a weakly viable E-inversive semigroup.

(iv) $S$ is an ideal extension of an idempotent free semigroup (possibly empty) by a semigroup $T$ which is the union of one idempotent semigroups with group ideals such that if $c, d \in T^{1}, a \in T$, cad and $c a^{2} d$ have a common component. 
ProOF. (i) $\Rightarrow$ (ii) follows from Theorem $\mathrm{i}$.

(ii) $\Rightarrow$ (i). Let $e \in \mathfrak{W}(c a d)$. Then $c a d x=\epsilon$ for some $x \in S$. Since $a$ and $a^{2}$ lie in the same component, $c a d x$ and $c a^{2} d x$ lie in the same component. Hence $e$ must be in the group ideal of this component. So, there exists $y \in S$ such that $c a^{2} d x y=e$. Hence $e \in c a^{2} d S$. Similarly $e \in S c a^{2} a$ and $\mathfrak{W}(c a d) \subseteq \mathfrak{W}\left(c a^{2} d\right)$. A similar argument shows $\mathfrak{B}\left(c a^{2} d\right) \subseteq \mathfrak{B}(c a d)$ and thus equality holds. Therefore $S$ is weakly viable.

(iii) $\Leftrightarrow$ (iv) follows from Propositicn 5 .

(i) $\Rightarrow$ (iii). Let $I=\left\{a \in S \mid S^{1} a S^{1} \cap E=\varnothing\right\}$. If $I=\varnothing, S$ is $E$-inversive by Lemma 3. Otherwise $I$ is an idempotent free ideal of $S$. $S / I$ is weakly viable by Lemma 2 and $E$-inversive since it has a zero.

(iii) $\Rightarrow$ (i) follows from Lemma 2 and Proposition 5 .

COROLlary. Let $S$ be viable. Then $S$ is weakly viable and if $\delta$ is defined by $a \delta b$ if and only if $\mathfrak{B}(a)=\mathfrak{W}(b)$, then $\beta=\delta$.

Proof. That $S$ is weakly viable follows from Theorem 6 of [3]. It is clear that $\beta \subseteq \delta$. Theorem 3 of [3] shows that $\delta$ satisfies (i) and (ii) of Theorem 1, so by (iii) of Theorem $1, \delta \subseteq \beta$. Hence $\delta=\beta$.

THEOREM 7. The foliowing are equivalent.

(i) $S$ is weakly viable and a power of each element lies in a subgroup.

(ii) $S$ is a band of semigroups which are ideal extensions of groups by nil semigroups.

Proof. (i) $\Rightarrow$ (ii). Let $B$ be any $\beta$-class and let $b \in B$. By the hypothesis there exists $e \in E$ and a positive integer $i$ such that $b^{i} \mathscr{H} e$. By Theorem 1 (iv), $b^{i} \beta e$. But $b^{i} \beta b$ and hence $e \in B$. Thus $B$ has a group ideal $K$ with $e \in K$. Since $b^{i} \mathscr{H} e, b^{i}=b^{i} e \in K$. Hence $B / K$ is nil. Therefore each $\beta$-class of $S$ is an ideal extension of a group by a nil semigroup and the result follows from Theorem 1.

(ii) $\Rightarrow$ (i) follows from Theorem 6 .

COROLlaRY. S is weakly viable and periodic (finite) if and only if $S$ is $a$ (finite) band of semigroups which are ideal extensions of periodic (finite) groups by (finite) nil semigroups.

A semigroup $S$ is separative if $x^{2}=x y=y^{2}(x, y \in S)$ implies $x=y$.

The proof of the following lemma is contained in the proof of Corollary 9 of [3].

LEMMA 8. Let $S$ be an ideal extension of a group by a nil semigroup. If $S$ is separative then $S$ is a group.

The equivalence of (ii) and (iii) in the following theorem was obtained by Clifford in [1] (or see [2, $\$ 4.2$, Exercise 3]). 
THEOREM 9. The following are equivalent.

(i) $S$ is weakly viable, separative and a power of each element lies in a subgroup.

(ii) $S$ is a band of groups.

(iii) For every $a, b \in S, a b S=a^{2} b S, S b a=S b a^{2}$ and $S$ is left and right regular.

(iv) $S$ is weakly viable, left and right regular.

(v) $S$ is weakly viable and regular.

(vi) $S$ is weakly viable and a union of groups.

Proof. (i) $\Rightarrow$ (ii). By Theorem $7, S$ is a band of semigroups which are ideal extensions of groups by nil semigroups. Since each one of these semigroups is separative, they are groups by Lemma 8 .

(ii) $\Rightarrow$ (iii) from [1, Theorem 7].

(iii) $\Rightarrow$ (iv) follows immediately from the observation that $S$ is weakly

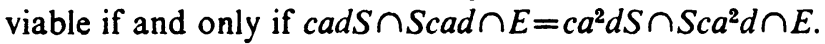

(iv) $\Rightarrow$ (v). By [2, Theorem 4.3], left and right regular imply regular.

(v) $\Rightarrow$ (vi). This will also follow from [2, Theorem 4.3] once we show that weakly viable and regular imply right regular. Suppose $a \in S$, and $a x a=a$. Now $\mathfrak{W}(a x)=\mathfrak{W}\left(a^{2} x\right)$ and $e=a x \in \mathfrak{W}(a x)$. Thus there exists $y \in S$ such that $a^{2} x y=e$. Hence $a^{2} x y a=e a=a$ and $S$ is right regular.

(vi) $\Rightarrow$ (i). A union of groups is always separative.

TheOREM 10. Suppose $a, b, c, d \in S, a b c d=e \in E$ implies that there exist $p, q \in S$ such that acp $=e, q b d=e$. Then $S$ is weakly viable. Hence every medial $(a b c d=a c b d)$ semigroup is weakly viable.

Proof. Let $a \in S$ and suppose $e \in \mathfrak{W}(c a d)$. Then there exist $u, v \in S$ such that $c a d u=e=v c a d$. Hence $(c a)(d u c)(a d) u=e$, so $(c a)(a d) p=e$. Similarly $q(c a)(a d)=e$. Hence $e \in \mathfrak{W}\left(c a^{2} d\right)$. A similar argument shows $\mathfrak{W}\left(c a^{2} d\right) \subseteq$ $\mathfrak{W}($ cad $)$. Hence $S$ is weakly viable.

It is immediate that medial semigroups satisfy the condition of the theorem.

We conclude with some problems for further study.

1 . Is a (weakly viable) $E$-inversive semigroup a disjoint union of semigroups with group ideals.

2. Characterize semigroups which are disjoint unions of semigroups having at most one idempotent.

3. Theorem 6 of [3] shows that a semilattice of semigroups having at most one idempotent is viable. Is a band of semigroups having at most one idempotent weakly viable?

4. Characterize those semigroups which are rectangular bands of semigroups having at most one idempotent. 
5. Study semigroup rings of weakly viabie, $E$-inversive semigroups. In this regard Weissglass has proved the following unpublished result: Let $D$ be a viable, $E$-inversive semigroup such that the only elements satisfying $x^{3}=x^{2}$ are idempotents. Let $R$ be a ring with no nil ideals. If the period of every element of finite order is prime to the characteristic of $R$ then the semigroup ring of $D$ over $R$ has no nil ideals.

One might be led to the conjecture that a weakly viable $E$-inversive semigroup is a band of one idempotent semigroups. In fact even a commutative $E$-inversive semigroup need not be such a band. Let $\langle a\rangle$ be the infinite cyclic semigroup generated by $a$ and let $T$ be the semigroup given by

\begin{tabular}{c|ccc} 
& 0 & $e_{1}$ & $e_{2}$ \\
\hline 0 & 0 & 0 & 0 \\
$e_{1}$ & 0 & $e_{1}$ & 0 \\
$e_{2}$ & 0 & 0 & $e_{2}$
\end{tabular}

Let $S=T \cup\langle a\rangle$ and define $t\langle a\rangle=\langle a\rangle t=t$ for every $t \in T$. Then $S$ is commutative and $E$-inversive, but it is easy to check that $S$ is not a band of one idempotent subsemigroups.

\section{REFERENCES}

1. A. H. Clifford, Bands of semigroups, Proc. Amer. Math. Soc. 5 (1954), 499-504. MR 15, 930.

2. A. H. Clifford and G. B. Preston, The algebraic theory of semigroups. Vol. 1 , Math. Surveys, no. 7, Amer. Math. Soc., Providence R.I., 1961. MR 24 \#A2627.

3. M. S. Putcha and J. Weissglass, A semilattice decomposition into semigroups having at most one idempotent, Pacific J. Math. 38 (1971).

Department of Mathematics, University of Calffornia, Santa Barbara, California 93106

Current address (Mohan S. Putcha): Department of Mathematics, University of California, Berkelev, California 94720 